\title{
Intracranial hemorrhage in full-term newborns: a hospital-based cohort study
}

\author{
Annemieke J. Brouwer • Floris Groenendaal • \\ Corine Koopman • Rutger-Jan A. Nievelstein • \\ Sen K. Han $\cdot$ Linda S. de Vries
}

Received: 4 January 2010 /Accepted: 30 March 2010/Published online: 15 April 2010

(C) The Author(s) 2010. This article is published with open access at Springerlink.com

\begin{abstract}
Introduction In recent years, intracranial hemorrhage (ICH) with parenchymal involvement has been diagnosed more often in full-term neonates due to improved neuroimaging techniques. The aim of this study is to describe clinical and neuroimaging data in the neonatal period and relate imaging findings to outcome in a hospital-based population admitted to a level 3 neonatal intensive care unit (NICU). Methods From our neuroimaging database, we retrospectively retrieved records and images of 53 term infants (1991-2008) in whom an imaging diagnosis of ICH with parenchymal involvement was made. Clinical data, including mode of delivery, clinical manifestations, neurological symptoms, extent and site of hemorrhage, neurosurgical intervention, and neurodevelopmental outcomes, were recorded.

Results Seventeen of the 53 term infants had infratentorial $\mathrm{ICH}, 20$ had supratentorial ICH, and 16 had a combination of the two. Seizures were the most common presenting symptom $(71.7 \%)$, another ten infants $(18.9 \%)$ presented with apneic seizures, and five infants had no clinical signs
\end{abstract}

A. J. Brouwer · F. Groenendaal · C. Koopman · L. S. de Vries $(\triangle)$ Department of Neonatology, Wilhelmina Children's Hospital, University Medical Center Utrecht,

PO Box 85090, 3508 AB Utrecht, The Netherlands

e-mail: 1.s.devries@umcutrecht.nl

R.-J. A. Nievelstein

Department of Radiology, Wilhelmina Children's Hospital, University Medical Center Utrecht,

Utrecht, The Netherlands

\section{S. K. Han}

Department of Neurosurgery, Wilhelmina Children's Hospital, University Medical Center Utrecht,

Utrecht, The Netherlands but were admitted to our NICU because of perinatal asphyxia $(n=2)$, respiratory distress $(n=2)$, and development of posthemorrhagic ventricular dilatation $(n=1)$. Continuous amplitude-integrated electroencephalography recordings were performed in all infants. Clinical or subclinical seizures were seen in 48/53 (90.6\%) infants; all received anti-epileptic drugs. Thirteen of all 53 (24.5\%) infants died. The lowest mortality rate was seen in infants with supratentorial ICH (10\%). Three infants with a midline shift required craniotomy, six infants needed a subcutaneous reservoir due to outflow obstruction, and three subsequently required a ventriculoperitoneal shunt. The group with poor outcome (death or developmental quotient (DQ) <85) had a significantly lower 5-min Apgar score $(p=.006)$. Follow-up data were available for 37/40 survivors aged at least 15 months. Patients were assessed with the Griffiths Mental Developmental Scales, and the mean DQ of all survivors was $97(\mathrm{SD}=12)$. Six infants $(17 \%)$ had a DQ below 85 [two of them had cerebral palsy (CP)]. Three infants developed CP (8.6\%); one had cerebellar ataxia, and two had hemiplegia.

Conclusion ICH with parenchymal involvement carries a risk of adverse neurological sequelae with a mortality of $24.5 \%$ and development of CP in $8.6 \%$. The high mortality rate could partly be explained by associated perinatal asphyxia. Infants with supratentorial ICH had a lower, although not significant, mortality rate compared with infants with infratentorial ICH and infants with a combination of supratentorial ICH and infratentorial ICH. In spite of often large intraparenchymal lesions, 30 of the 34 survivors without CP $(88.2 \%)$ had normal neurodevelopmental outcome at 15 months.

Keywords Intracranial hemorrhage $\cdot$ Intraparenchymal hemorrhage $\cdot$ Subdural hemorrhage $\cdot$ Full-term newborns 


\section{Introduction}

Neonatal intracranial hemorrhage (ICH) has five major clinical types: subdural hemorrhage (SDH), primary subarachnoid hemorrhage $(\mathrm{SAH})$, cerebellar hemorrhage, intraventricular hemorrhage (IVH), and intraparenchymal hemorrhage. SDH and intraparenchymal hemorrhage are more often found in the term infant, while SAH, IVH, and cerebellar hemorrhage are more common in the preterm infant [1].

$\mathrm{ICH}$, including SDH (with or without parenchymal involvement), used to be a common cause of neonatal death following a traumatic delivery, but the incidence has decreased with improved obstetric care. In recent years, SDH has been recognized more often mainly because neuroimaging is now also performed routinely in infants not presenting with clinical symptoms [1]. ICH in the term infant usually presents with nonspecific neurological signs and symptoms. Signs can be general and may not point directly to the brain as the source of the problem [2].

$\mathrm{ICH}$ in the newborn is frequently associated with prolonged or precipitous delivery, vaginal breech delivery, instrumental delivery, use of forceps or ventouse extraction, and primiparity or extreme multiparity [2-6]. Hemorrhage may be due to rupture of veins in the subdural space, with bleeding from the venous sinus or from hemorrhage within the cerebellum [2]. Accumulation of blood in the posterior fossa can cause neurological symptoms that manifest within the first few days after delivery, such as a tense or bulging fontanelle, increasing head circumference, apneas, bradycardia, and/or seizures [3]. The true incidence of ICH is probably higher than reported because only a fraction of the infants with ICH present with clinical symptoms [1].

The aim of this study was to describe precipitating factors, presenting symptoms, neuroimaging data, and neurodevelopmental outcome of infants in whom an imaging diagnosis [computed tomography (CT) or magnetic resonance imaging (MRI)] of ICH was made.

\section{Methods and patients}

\section{Patients}

The study was approved by the local ethical committee. Between October 1991 and November 2008, 53 neonates with a gestational age (GA) of 37 weeks or more were admitted to the level 3 neonatal intensive care unit (NICU) of a hospital with a diagnosis of ICH associated with parenchymal involvement. All records and images of infants in whom an imaging diagnosis of an ICH was made were retrieved from a neuroimaging database. Clinical data, including mode of delivery, clinical manifestations, neurological symptoms, treatment, neurosurgical intervention, and neurodevelopmental outcomes, were recorded. Infants were only eligible when SDH or SAH was diagnosed in the presence of associated parenchymal injury. Neurological development was assessed in all surviving patients at approximately 24 months of age.

\section{Neuroimaging}

Cranial ultrasound was performed within the first $6 \mathrm{~h}$ of admission and repeated at least two times a week until discharge.

Ultrasound findings were confirmed by CT and/or MRI. $\mathrm{CT}$ was only performed on the day of admission when a shift in midline was noted with cranial ultrasound and neurosurgical intervention was considered. MRI was the preferred neuroimaging technique and was performed within a week of delivery to obtain detailed information about parenchymal involvement.

MRI was performed on a 1.5-T Philips system (Intera or Achieva 1.5 T; Philips, Healthcare, Best, The Netherlands). Sagittal $T_{1}$-weighted images $\left(T_{\mathrm{R}}=450-550 \mathrm{~ms} ; T_{\mathrm{E}}=15-30 \mathrm{~ms}\right.$; slice thickness $=5 \mathrm{~mm}$ ), axial inversion recovery (IR) scan $\left(T_{\mathrm{R}}=2,500-3,000 \mathrm{~ms} ; T_{\mathrm{E}}=30 \mathrm{~ms}\right.$; slice thickness $=2$ or $5 \mathrm{~mm})$, and axial $T_{2}$-weighted images $\left(T_{\mathrm{R}}=3,000-3,150 \mathrm{~ms}\right.$; $T_{\mathrm{E}}=150 \mathrm{~ms}$; slice thickness $=2-5 \mathrm{~mm}$ ) were acquired. During the last 4 years, phase-contrast angiography (PCA) images have been added to the protocol. When indicated, magnetic resonance venography (MRV) was performed with flow velocities of 30 and $15 \mathrm{~cm} / \mathrm{s}$. PCA images became available in 2003 but were used routinely starting 2006, and threedimensional (3D) MRV became available in 2008. Since 1995, diffusion-weighted (DW) MRI has been performed using an interweaved DW acquisition with an echo planar imaging factor of 41 , a $T_{\mathrm{R}} / T_{\mathrm{E}}$ of $4,000 / 148 \mathrm{~ms}$, a field of view of $210 \mathrm{~mm} \times 210 \mathrm{~mm}$, a slice thickness of $4 \mathrm{~mm}$, a slice gap of $0.5 \mathrm{~mm}$, and $b$ factors of 0 and $1,000 \mathrm{~s} / \mathrm{mm}^{2}$. After completion of imaging, the trace apparent diffusion coefficient map was calculated to avoid confusion with $T_{2}$ effects.

ICH was divided into three categories based on the site of hemorrhage; supratentorial, infratentorial, or a combination of the two sites. Every lobe (frontal, temporal, parietal, and occipital) of every case was described in detail. The presence of a midline shift was recorded.

\section{Amplitude-integrated electroencephalography}

Continuous one-channel or two-channel amplitudeintegrated electroencephalography (aEEG) recordings were routinely used in all infants admitted with perinatal asphyxia or neurological problems (such as Apgar score at $5 \mathrm{~min}<5, \mathrm{pH}<7.10$, multiorgan failure, or clinical seizures) to assess the background pattern and to aid in the detection of (sub)clinical seizures. 
Anti-epileptic medication protocol

Phenobarbital is the first choice for anti-epileptic drug (AED). From 1992 to 1998, lidocaine was used as rescue treatment when other anti-convulsive drugs (phenobarbital, phenytoin, and midazolam) had failed [4]. Lidocaine is administered following a strict protocol. From 1998 onwards, we have been using a strict AED protocol: phenobarbital first, followed by midazolam and lidocaine.

Assessment of neurodevelopmental outcome

The surviving infants were seen in the follow-up clinic at regular intervals. Assessment of outcome was based on clinical examination at discharge and follow-up using the Griffiths Mental Developmental Scales (GMDS) at a mean age of 20 months.

The GMDS provides a subscale and general developmental age and quotient equivalents (mean $=100 ; \mathrm{SD}=15)$ of raw scores. Five domains of functioning are tested: locomotor, personal-social, hearing and speech, eye-hand coordination, and performance. The GMDS shows continuity validity with time and across cultures [5, 6]. A DQ of $>85$ at 2 years of age is considered to be within the reference range. The infants were assessed by developmental pediatricians/neonatologists using the GMDS.

\section{Statistics}

Statistical analysis was performed using the Mann-Whitney or Kruskal-Wallis test, the chi-square test for comparison of proportions, and analysis of variance for comparison of means. Statistical analyses were performed using the SPSS software for Windows, version 15 (SPSS, Chicago, IL). Statistical significance was set at $p=.05$.

\section{Results}

\section{Clinical characteristics}

Between October 1991 and November 2008, 53 full-term neonates (30 male neonates and 23 female neonates) with ICH and associated parenchymal involvement were admitted to our level 3 NICU. All infants had both intraaxial hemorrhage (intraparenchymal and intraventricular) and extra-axial hemorrhage (subdural and subarachnoid). Associated SDH was present in 50/53 infants (94.3\%), SAH was present in $8 / 53$ infants $(15.1 \%)$, and associated IVH was present in $12 / 53$ infants $(22.6 \%)$. A combination of SAH and SDH was present in 6/53 infants $(11.3 \%)$; from among these six infants, three $(5.7 \%)$ had an IVH as well.
The clinical characteristics of the infants, divided into three groups (supratentorial, infratentorial, and supratentorial plus infratentorial), are summarized in Table 1. Clinical presentation occurred in $47.2 \%$ on the first day after birth $(n=25)$ and in $26.4 \%$ on the second day $(n=14)$; eight infants showed signs between 3 and 5 days, and one infant first presented with severe ventricular dilatation at 30 days. Seizures were the most common presenting symptom $(71.7 \%)$; a further ten infants $(18.9 \%)$ presented with apneic seizures, and five infants had no clinical signs but were admitted to our NICU because of perinatal asphyxia $(n=2)$, respiratory distress $(n=2)$, and development of posthemorrhagic ventricular dilatation $(n=1)$.

Infants with poor outcome (death or a DQ of $<85$ ) had a significantly lower Apgar score at $5 \mathrm{~min}(p=.006)$. All were born at term ( $>37$ weeks), with a mean birth weight of $3,265 \mathrm{~g}(\mathrm{SD}=630) ; 8$ infants $(15.1 \%)$ had a birth weight of $\geq$ p95, and 12 infants $(22.6 \%)$ had a birth weight below p10, according to The Dutch Perinatal Registry Birth Weight centiles (http://www.perinatreg.nl/referentiecurven).

Thrombocytopenia $\left(<150 \times 10^{9} \mathrm{~L}^{-1}\right)$ was present in $11 / 50$ (22\%; data for three infants were not available). Thrombocytopenia was significantly more common in infants who died than in survivors (6/13 versus 5/37; $p=.023)$. Thrombocytopenia was due to neonatal alloimmune thrombocytopenic purpura in one infant.

Among 9 of 53 infants, clotting time (activated partial thromboplastin time and thrombin time) was not available. Of the remaining 44 infants, $12(27.3 \%)$ infants had prolonged clotting time; 5 of 32 survivors (missing data for eight) had prolonged clotting time versus 7 of 12 infants who died (missing data for one) $(p=.008)$. One of the survivors had hemophilia A (factor VIII).

\section{Mode of delivery}

Thirty-six of the 53 mothers $(67.9 \%)$ were nulliparous (Table 2). Uncomplicated spontaneous vaginal delivery was the mode of delivery in $14 / 53(26.4 \%)$. In 10/20 (50\%) infants with supratentorial ICH compared to $2 / 17(11.8 \%)$ infants with infratentorial ICH and 2/16 (12.5\%) infants with supratentorial and infratentorial $\mathrm{ICH}$, an uncomplicated spontaneous vaginal delivery was the mode of delivery $(p=.010)$. Twelve infants $(22.6 \%)$ were delivered with ventouse extraction; $7 / 17(41.2 \%)$ infants were delivered with infratentorial ICH compared to $3 / 20$ (15\%) infants with supratentorial ICH and 2/16 infants (12.5\%) with supratentorial and infratentorial ICH $(p=.174)$. Vaginal breech delivery was the mode of delivery in $11 / 56(25 \%)$, in $3 / 17(17.6 \%)$ infants with infratentorial $\mathrm{ICH}$, and in $8 / 16$ $(50 \%)$ infants with supratentorial and infratentorial $\mathrm{ICH}$ $(p=.132)$. 
Table 1 Clinical characteristics of the study population.

\begin{tabular}{lllll}
\hline & Supratentorial $(n=20)$ & Infratentorial $(n=17)$ & Supratentorial + infratentorial $(n=16)$ & $P$ \\
\hline Male/female infants & $9 / 11$ & $11 / 6$ & $10 / 6$ & .411 \\
GA (weeks) [mean $\pm \mathrm{SD}]$ & $38 \pm 1$ & $40 \pm 1.2$ & $39 \pm 1.3$ & .005 \\
Birth weight $(\mathrm{g})[$ mean $\pm \mathrm{SD}]$ & $3,229 \pm 719$ & $3,437 \pm 539$ & $3,130 \pm 594$ & .378 \\
Apgar score at 1 min [median (range)] & $8(0-10)$ & $6(0-10)$ & $7(1-9)$ & .046 \\
Apgar score at 5 min [median (range)] & $10(4-10)$ & $6(0-10)$ & $8(1-10)$ & .033 \\
Day of clinical presentation [median (range)] & $2(1-4)$ & $1(1-5)$ & $1(1-30)$ & .096 \\
Day of MRI/CT [median (range)] & $1(1-9)$ & $2(1-14)$ & $1(1-26)$ & .77 \\
Mortality [n $(\%)]$ & $2(10)$ & $6(35.3)$ & $5(31.3)$ & .160 \\
\hline
\end{tabular}

Of the five infants delivered by Caesarean section (CS), four were delivered by emergency $\mathrm{CS}$, and one was delivered by elective CS because of breech presentation.

\section{Clinical findings}

Thirty-three (62.3\%) infants required intubation and ventilation: 31 infants were intubated due to respiratory insufficiency, and 2 infants were intubated and ventilated prior to neurosurgical intervention.

aEEG recordings were obtained in all infants. Clinical or subclinical seizures were the presenting symptom in $48 / 53$ (90.6\%) infants, and different AEDs were administered to all. Phenobarbital was administered to 46 infants, 1 infant received lidocaine, and 1 infant showed seizures before admission and needed no medication after admission. Of these 48 infants, 18 were treated with phenobarbital monotherapy, 12 infants needed two different AEDs, and 18 infants needed three or more AEDs. Of the 13 infants who died, 1 infant had status epilepticus, 7 infants had an isoelectric recording, 4 infants had a burst-suppression background pattern with epileptic activity, and 1 infant had a discontinuous background pattern without sleep-wake cycling. In all but four infants, a standard electroencephalogram was performed as well to confirm the aEEG diagnosis.

\section{Mortality}

Intensive care was withdrawn in 13 of the 53 infants (24.5\%) because of a combination of neuroimaging findings and neurophysiologic data, consisting mostly of a severely abnormal aEEG result, including a very suppressed background pattern with seizures that persisted despite administration of different AEDs. Infants with supratentorial ICH had a lower, although not significant, mortality rate compared with infants with infratentorial $\mathrm{ICH}$ and infants with both supratentorial and infratentorial $\mathrm{ICH}$; 2/20 (10\%) infants with supratentorial ICH, 6/17 (35.3\%) infants with infratentorial $\mathrm{ICH}$, and 5/16 (31.3\%) infants with $\mathrm{ICH}$ involving both the supratentorial region and the infratentorial region died.

Table 2 Delivery.

\begin{tabular}{|c|c|c|c|c|}
\hline Delivery & Supratentorial $(n)$ & Infratentorial $(n)$ & Supratentorial + infratentorial $(n)$ & Total $(n)$ \\
\hline Breech & & 3 & 8 & 11 \\
\hline Vaginal & 10 & 2 & 2 & 14 \\
\hline Ventouse & 3 & 7 & 2 & 12 \\
\hline Ventouse followed by CS & 1 & 2 & 1 & 4 \\
\hline Precipitous & 1 & 1 & & 2 \\
\hline Forceps/breech & & 1 & & 1 \\
\hline Ventouse + forceps followed by CS & & 1 & & 1 \\
\hline $\mathrm{CS}$ & 4 (1 elective) & & 1 & 5 \\
\hline Ventouse + forceps & & & 1 & 1 \\
\hline Forceps & & & 1 & 1 \\
\hline Prolonged & 1 & & & 1 \\
\hline Total & 20 & 17 & 16 & 53 \\
\hline
\end{tabular}




\section{Postmortem examination}

In $10 / 13$ infants, parental permission for autopsy was obtained. In four infants, infratentorial SDH was associated with a tentorial tear. Three infants had parenchymal hemorrhage associated with extensive ischemic lesions, one infant had massive ICH, one infant had massive hemorrhage in the temporal lobe combined with parenchymal infarction, and one infant had temporal parietal hemorrhage associated with parenchymal cysts and histological findings compatible with hypoxic ischemia following neonatal alloimmune thrombocytopenia.

\section{Imaging findings}

All infants were examined with cranial ultrasound. MRI or CT was performed in all but four infants, who were too unstable to be transported to the CT or MRI unit. Twelve infants had CT only, 12 infants first had CT followed by MRI, and 24 infants had MRI only. Two of the four infants who did not have MRI or CT had postmortem examination.

The frontal lobe was predominantly affected in 11 infants; this was associated with a midline shift in 7 infants and required surgical intervention in 3 infants (Fig. 1). Delivery was uncomplicated in $7 / 11$ infants $(63.6 \%)$. The temporal lobe was primarily involved in ten infants. Both frontal and temporal parenchymal hemorrhages can be associated with cerebral sinovenous thrombosis (CSVT). Only 4/21 infants with frontal or temporal parenchymal hemorrhage, possibly associated with CSVT, were studied with PCA, but none had 3D MRV. A possible CSVT of the superior sagittal sinus was suspected on PCA in one child with a large frontal parenchymal hemorrhage.

The occipital lobe was more often involved in infants with both supratentorial and infratentorial hemorrhage
Fig. 1 CT performed on day 2 (a, b), showing large intraparenchymal hemorrhage and SDH in the right frontal lobe, causing a shift in midline. MRI and IR axial view obtained on day 8 following craniotomy (c) showing resolution of the midline shift and reduction of subdural and intraparenchymal hematoma. A repeat MRI and IR axial view at 3 months (d) shows a small area of cavitation and mild atrophy of the right frontal lobe. Outcome was well within the normal range at 2 years of age
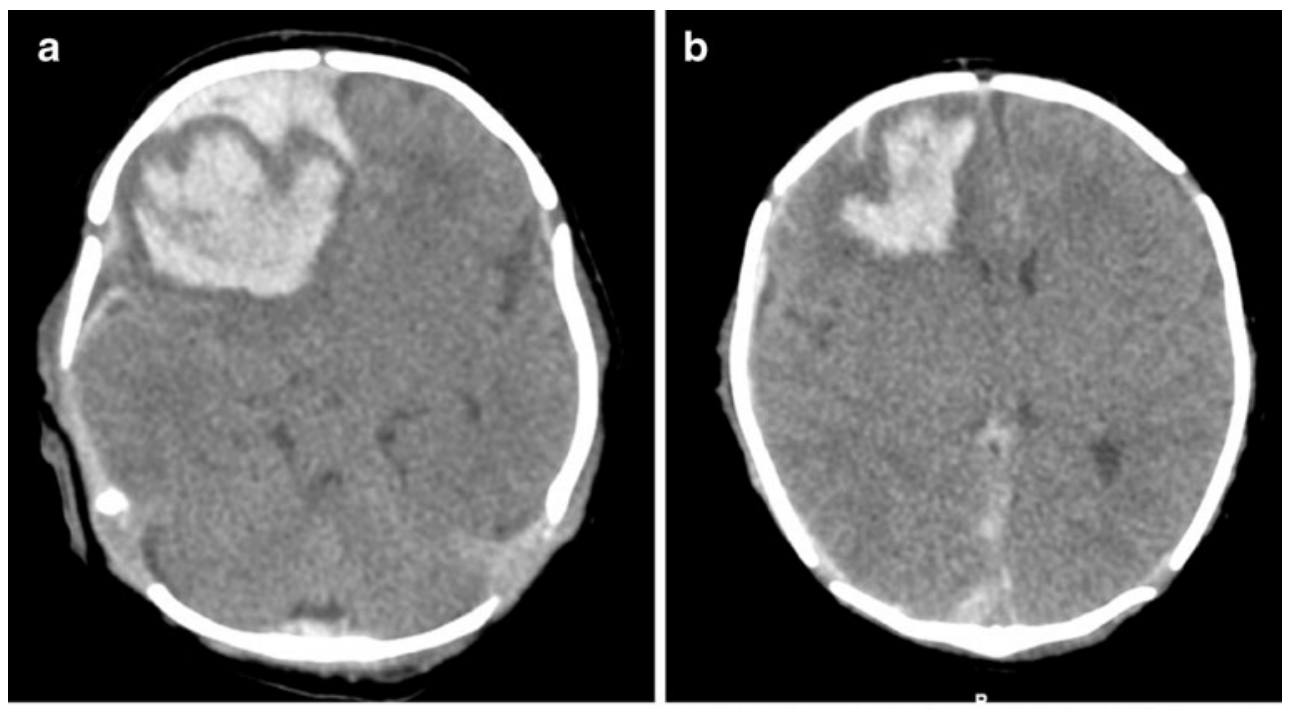

C

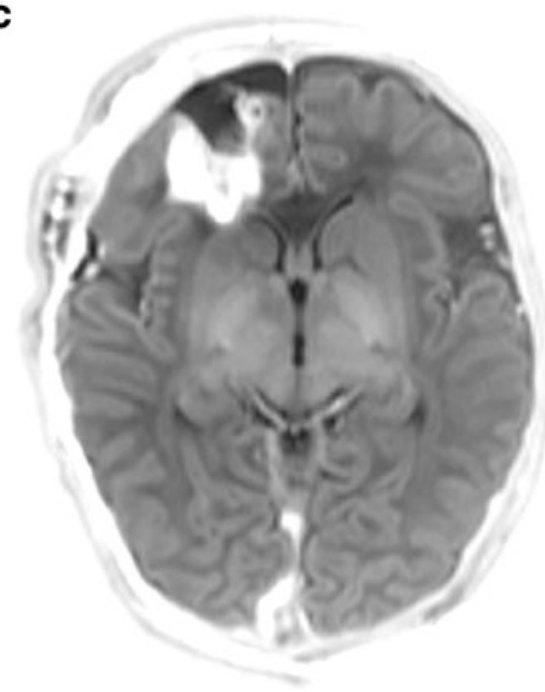

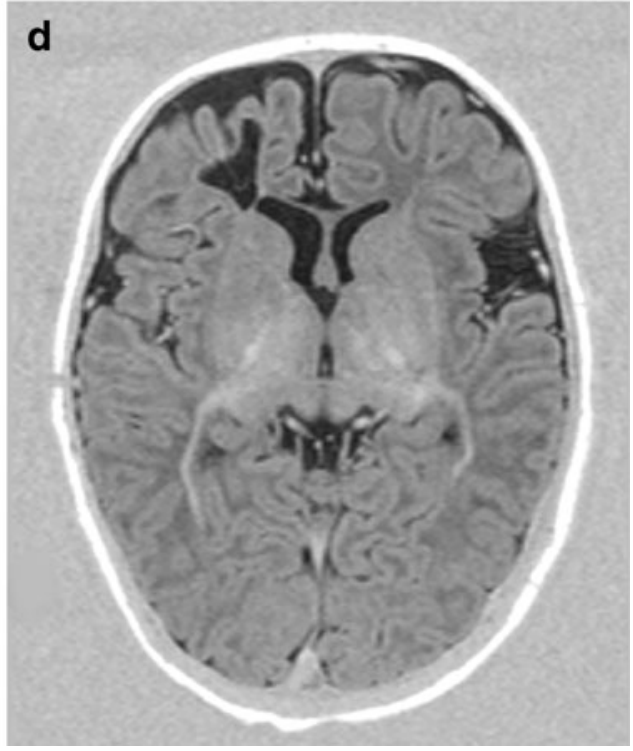


(75\%), compared with $40 \%$ of the infants with supratentorial hemorrhage (Fig. 2).

Follow-up MRI was performed in 30/40 survivors between 6 weeks and 10 years.

\section{Surgical intervention}

Twenty-one infants (39.6\%) showed an associated midline shift. In only three infants was this considered severe enough to require neurosurgical intervention (all three had supratentorial ICH). These infants had craniotomy to reduce intracranial pressure. Of the 21 infants, 12 (57.1\%) had supratentorial ICH. In the infratentorial ICH group, $5 / 17(29.4 \%)$ showed a midline shift of the cerebellum. Of the infants with supratentorial and infratentorial $\mathrm{ICH}$, four $(25 \%)$ had a midline shift, of which one infant had both a supratentorial midline shift and a midline shift of the cerebellum.

Sixteen (30.2\%) infants developed hydrocephalus due to outflow obstruction following supratentorial hemorrhage in $3 / 20$, infratentorial hemorrhage in $7 / 17$, and combined supratentorial and infratentorial hemorrhage in 6/16. Nine infants were treated with isosorbide, an osmotic diuretic that is considered to reduce the production of cerebrospinal fluid [7]. Six infants had insertion of a subcutaneous ventricular reservoir to control ventricular size and intraventricular pressure, which was increased due to outflow obstruction; one infant had an external ventricular drain. Three infants subsequently needed a ventriculoperitoneal drain; in two infants, revision of the ventriculoperitoneal drain was necessary, and one infant needed multiple revisions.
Outcome

Of the 40 surviving infants, 37 were seen in the follow-up clinic at a mean age of 20 months (range, 15-29 months of age; 24 infants were between 18 and 26 months of age); one infant was lost to follow-up, and two infants were still too young. The mean DQ was $97(\mathrm{SD}=12)$. Infants with infratentorial ICH had a mean DQ of $99(\mathrm{SD}=12)$, those with supratentorial ICH had a mean DQ of $96(\mathrm{SD}=11)$, and those with supratentorial and infratentorial ICH had a mean DQ of $96(\mathrm{SD}=14)$, which was not significantly different. Of these 37 infants, $31(83.8 \%)$ had a DQ of $>85$. No significant difference in outcome - whether or not a midline shift was present - was found. Infants needing neurosurgical intervention, either craniotomy or reservoir, had a mean DQ of 96 $(\mathrm{SD}=13)$. No significant difference was found on whether or not the infant needed neurosurgery.

Of the 12 infants with a birth weight below p10, seven had poor outcome (four infants died, and three had a DQ of $<85$ ), and three infants had a DQ of $>85$ (missing data for one, and one was too young). Six of the eight infants with a birth weight of $\geq$ p95 had a DQ of $>85$, and one infant died.

Involvement of a single lobe was seen in 9 of 20 infants with supratentorial ICH. No significant difference in outcome was seen on whether or not there was involvement of one, two, or three lobes. The infants in which the frontal lobe was predominantly affected had a mean $\mathrm{DQ}$ of $99(\mathrm{SD}=8.3)$, and one infant had a DQ of $<85$ (missing data for two).

One infant with supratentorial ICH developed postneonatal epilepsy. Two infants - one with infratentorial $\mathrm{ICH}$ and one with supratentorial and infratentorial $\mathrm{ICH}-\mathrm{had}$
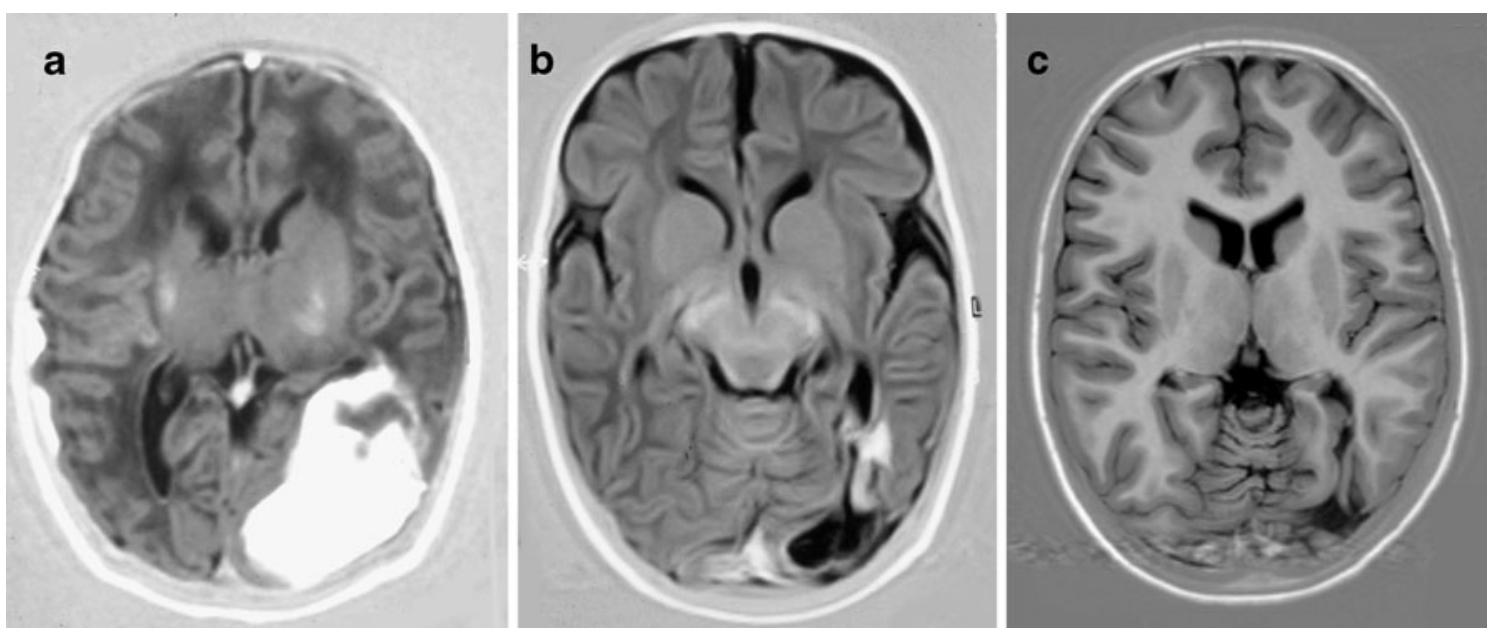

Fig. 2 MRI and IR axial view obtained on day 8 , at 3 months, and at 11 years. A large parieto-occipital hemorrhage is seen in the neonatal period (a). The hemorrhage has almost completely resolved by
3 months, resulting in a small cavity (b), which is still present at 11 years $(\mathbf{c})$. Outcome is within the normal range 
visual impairment. One infant with supratentorial and infratentorial $\mathrm{ICH}$ had infantile esotropia. Three infants developed cerebral palsy (CP); one infant developed cerebellar ataxia following a severe cerebellar hemorrhage (Fig. 3) (never achieved independent walking), and two infants developed hemiplegia following severe supratentorial hemorrhage; one needed craniotomy, and one scored low on all five domains of the GMDS.

\section{Discussion}

We report neonatal and follow-up data of 53 full-term infants with ICH with associated parenchymal involvement admitted to a level 3 NICU over a period of 17 years. This is a large hospital-based cohort of infants with symptomatic ICH. Most studies [1, 8-10] described asymptomatic infants with SDH or small series of symptomatic ICH [11-14]. The study of Rooks et al. [9] showed a high incidence of ICH in asymptomatic infants.
In our study, the mortality rate was high $(24.5 \%)$, but the neurodevelopmental outcome among the survivors, with known outcome, was within the normal range in the majority of the children $(83.8 \%)$. The high mortality rate could partly be explained by associated perinatal asphyxia, since the group with poor outcome (death or a DQ of $<85$ ) had a significantly lower Apgar score at $5 \min (p=.006)$. Apgar scores at $1 \mathrm{~min}$ were less than 7 in $46.2 \%$ of the infants, and Apgar scores at 5 min were less than 7 in $28.8 \%$ of the infants. Infants with poor outcome had a significantly lower Apgar score at $5 \mathrm{~min}$, which could be due to associated perinatal asphyxia.

The most important finding in the report by Towner et al. [15] is that forceps assistance, ventouse extraction, and CS were all associated with an increased risk for ICH. Benedetti [16] stated that if an attempt for operative vaginal delivery fails, the risk of injury is increased no matter which method of delivery is chosen. In the study of Whitby et al. [1], those delivered by forceps after attempted ventouse delivery were more likely to have SDH compared
Fig. 3 MRI, $T_{1}$-weighted spinecho, midsagittal view (a), and IR, axial view (b), both obtained on day $10 ; T_{1}$-weighted spinecho, midsagittal view (c), and IR, axial view, obtained at 18 months of age $(\mathbf{c}, \mathbf{d})$. A large infratentorial hemorrhage involving the left cerebellar hemisphere and the fourth ventricle is seen. There is also supratentorial SDH (a, b). Follow-up MRI shows severe atrophy of the vermis and the left cerebellar hemisphere (c, d). The child developed severe ataxia and also has growth hormone deficiency
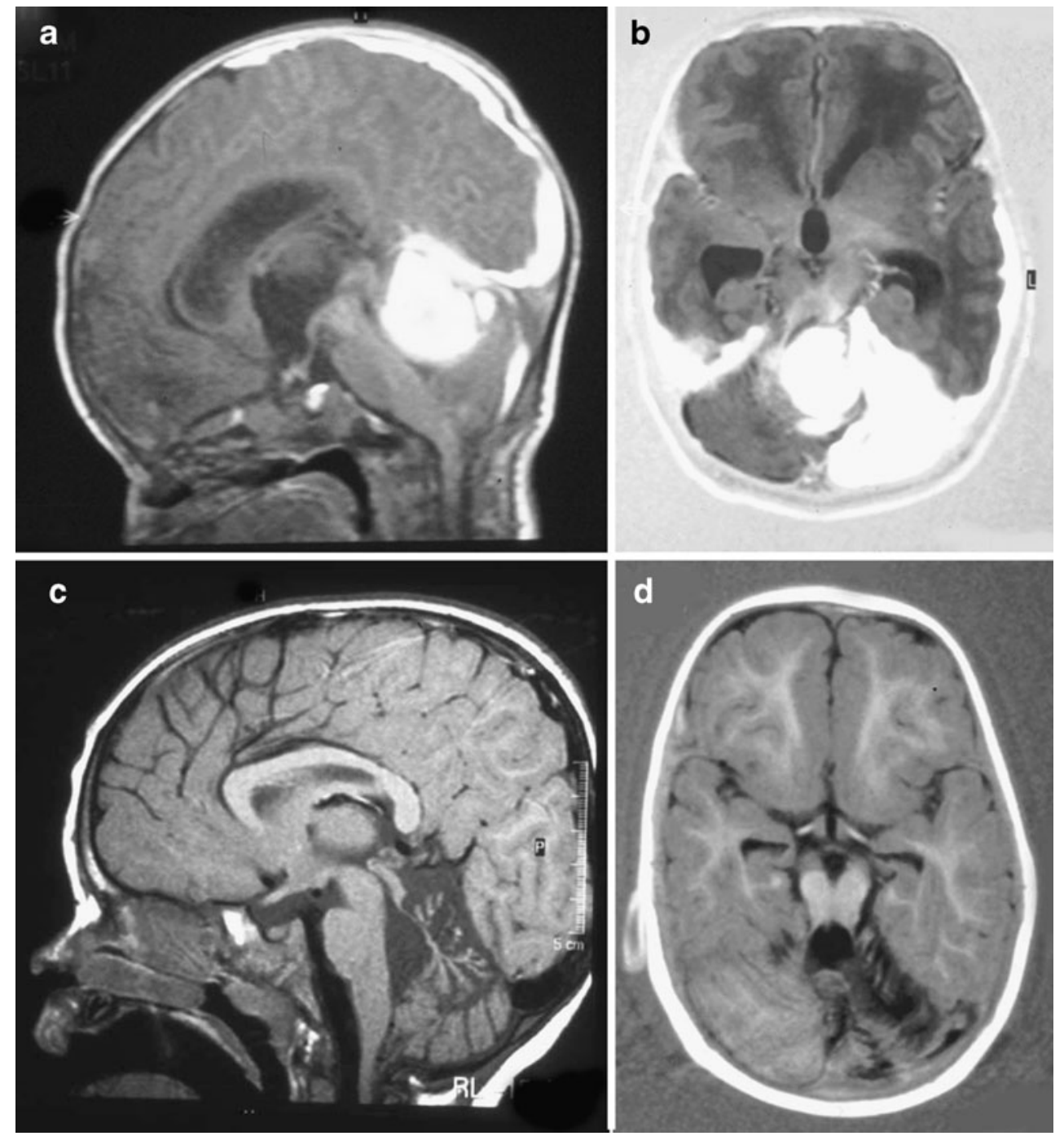
to those delivered by any other method of delivery. The common risk factor for ICH is therefore complicated labor $[15,17]$. This is similar to the results in our population, where $38 \%$ of the deliveries were assisted ventouse or forceps deliveries or CS following a failed attempt for instrumental vaginal delivery. Fifteen infants $(27 \%)$ in our population were delivered by elective CS $(n=1)$ or were uncomplicated vaginal deliveries $(n=14)$; all these infants had supratentorial ICH. Huang and Robertson [12] also reported five infants who had spontaneous parenchymal hemorrhage after a vaginal delivery. The series of Looney et al. [8] and Whitby et al. [1] did not report any infant with ICH after delivery through CS. In our group, however, five infants with supratentorial ICH were delivered by CS; four infants were delivered via emergency CS because of fetal distress.

Several risk factors have been reported in term newborns with ICH, but only a few studies have demonstrated a relationship between the proposed risk factors and $\mathrm{ICH}$, and these comprise only a small number of cases [18]. Thrombocytopenia is the most common condition associated with ICH [19]. In our population, 11 infants (22\%) had a platelet count of less than $150 \times 10^{9} \mathrm{~L}^{-1}$ on admission (data on three infants are missing), with $8 \%$ of the infants having a platelet count of $<70 \times 10^{9} \mathrm{~L}^{-1}$. Jhawar et al. [19] described a high prevalence $(30.8 \%)$ of infants with a platelet count of $<70 \times 10^{9} \mathrm{~L}^{-1}$, and they concluded that thrombocytopenia is the most important predictor of $\mathrm{ICH}$
Fig. 4 MRI, IR (a, b), and $T_{2}$ spin-echo (c) axial views obtained on day 10 , at 3 months, and at 7 years, and coronal IR (d) obtained at 7 years. A large hemorrhage in the temporal lobe is seen in the neonatal period (a), with cystic evolution at 3 months (b). End stage of the lesion showing complete destruction of the left temporal lobe can be appreciated at 7 years $(\mathbf{c}, \mathbf{d})$. Outcome is complicated by behavioral problems (tics and hyperactivity)
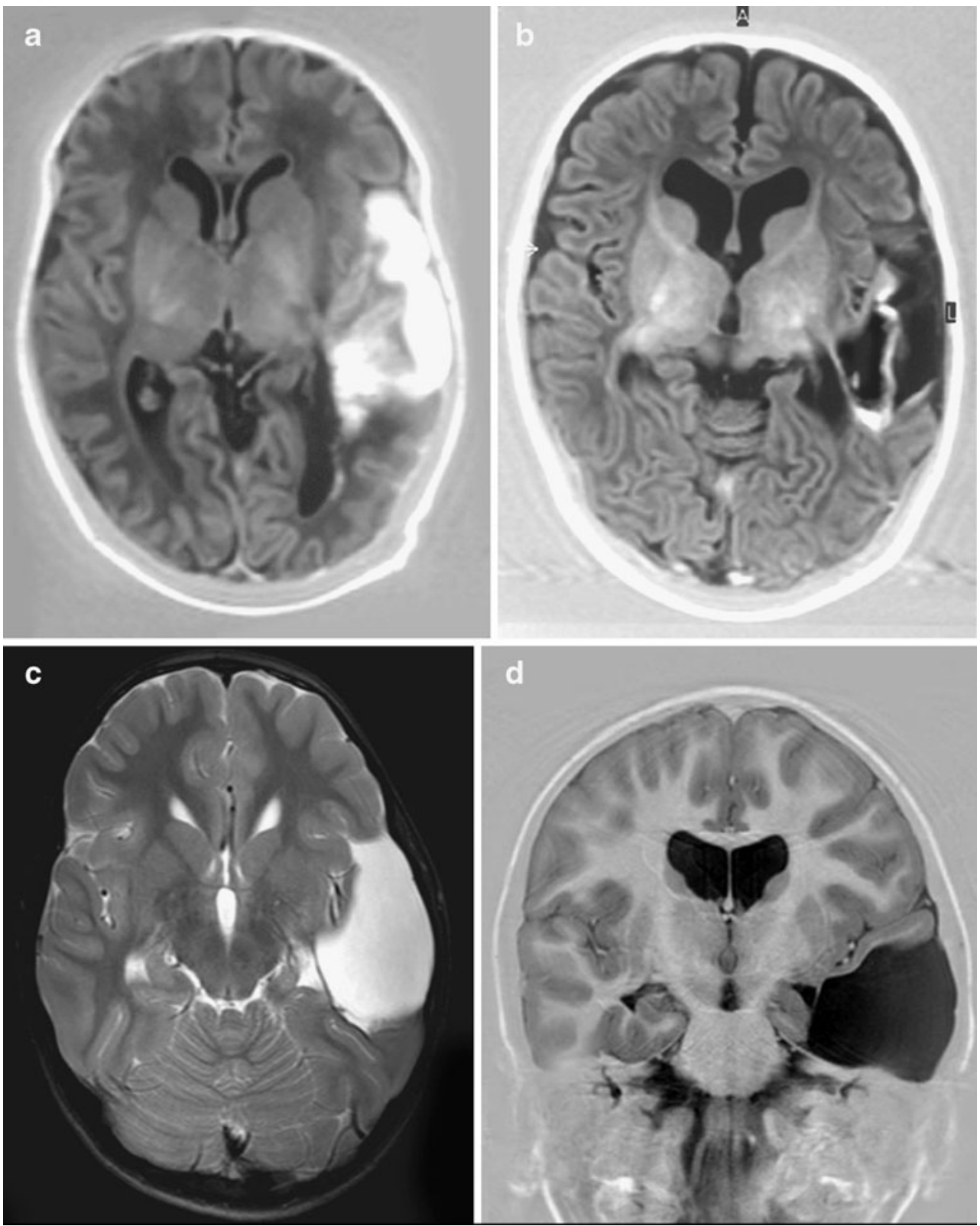
and is also associated with the most severe type of hemorrhage, in agreement with our findings. Infants who died had significantly more often thrombocytopenia [19]. It is therefore recommended that a coagulation profile be obtained from all infants suspected to have ICH. Since this is a retrospective study, we have limited data about the associated coagulopathy of our infants.

The mean birth weight in our group was on the 40th percentile; however, 9 infants (17\%) had a birth weight above the 90th percentile, and 12 infants $(22.6 \%)$ had a birth weight below the 10th percentile. Infants with a birth weight of $<10$ th percentile were more at risk for an adverse outcome. We did not have reliable head circumference data on our patients because caput succedaneum was often present following a ventouse extraction. This measurement was therefore not included in the analysis.

Nulliparous women are more likely to deliver with ventouse or forceps assistance and therefore are at increased risk [17, 20]. In our cohort, $36(67.9 \%)$ of the mothers were nulliparous, and only one woman was an extreme multiparous, an associated factor previously mentioned by others [21].

While the strength of this study is that it spans a period of almost 20 years, this also led to a major limitation of this study, with a change in imaging sequences over the years. While we were initially only able to perform $T_{1}$ and $T_{2}$ sequences in these newborn infants, diffusion-weighted imaging became available in the mid-1990s; MRV (initially phase-contrast parasagittal scans and later 3D MRV) became available some 4 years ago, enabling us to diagnose CSVT in some of these children, while this could only be assumed in those born in the 1990s (Fig. 4). Infants with a confirmed CSVT with unilateral thalamic hemorrhage were reported previously [22]. We consider this subgroup of infants as a different group - often displaying a later onset of symptoms and mostly born after an uncomplicated vaginal delivery, but with signs of infection and/or dehydration - and therefore did not include them in the present study [23]. It is well possible that our infants with temporal lobe hemorrhage did indeed have sinovenous thrombosis, but we were unable to prove this due to lack of MRV in most of these infants. It has recently been suggested that infants with temporal lobe hemorrhage tend to present with apneic spells $[24,25]$. In our group, 11 infants had involvement of the temporal lobe, and 6/11 $(54.5 \%)$ presented with apneic seizures (Fig. 4) Slaughter et al. [13] described the surprisingly good outcome of infants with hemorrhagic infarct in the temporal lobe. As there was only one infant with a single temporal hemorrhage in our population, we cannot draw the same conclusion. Most of our infants also had involvement of either the parietal lobe and/or the occipital lobe. However, our conclusion for this whole population is the same: despite often impressive imaging abnormalities and clinical presentation, infants with ICH tend to have better early outcome than expected. Clinical follow-up needs to be continued to school age, as these infants are likely to be at risk for cognitive or behavioral problems at school age.

\section{Conclusion}

ICH with parenchymal involvement carries a risk of adverse neurological sequelae with a mortality of $24.5 \%$ and a development of $\mathrm{CP}$ in $8.6 \%$. The high mortality rate could partly be explained by associated perinatal asphyxia. Infants with supratentorial ICH had a lower, although not significant, mortality rate compared with infants with infratentorial ICH and infants with combined supratentorial and infratentorial ICH. In spite of often large intraparenchymal lesions, $88.2 \%$ of the survivors without CP had a normal early neurodevelopmental outcome.

Conflict of interest statement We declare that we have no conflict of interest.

Open Access This article is distributed under the terms of the Creative Commons Attribution Noncommercial License which permits any noncommercial use, distribution, and reproduction in any medium, provided the original author(s) and source are credited.

\section{References}

1. Whitby EH, Griffiths PD, Rutter S, Smith MF, Sprigg A, Ohadike $P$ et al (2004) Frequency and natural history of subdural haemorrhages in babies and relation to obstetric factors. Lancet 363(9412):846-851

2. Levene M (2005) Part 3. Intracranial haemorrhage at term. In: Rennie JM (ed) Roberton's textbook of neonatology, 4th edn. Elsevier, Philadelphia, pp 1120-1128

3. Perrin RG, Rutka JT, Drake JM, Meltzer H, Hellman J, Jay V et al (1997) Management and outcomes of posterior fossa subdural hematomas in neonates. Neurosurgery 40(6):1190-1199, discussion $1199-1200$

4. van Rooij LG, Toet MC, Rademaker KM, Groenendaal F, de Vries LS (2004) Cardiac arrhythmias in neonates receiving lidocaine as anticonvulsive treatment. Eur J Pediatr 163(11):637-641

5. Griffiths R (1976) The abilities of babies. A study in mental measurement. Association for Research in Infant and Child Development, Amersham, Great Britain

6. Luiz DM, Foxcroft CD, Stewart R (2001) The construct validity of the Griffiths Scales of Mental Development. Child Care Health Dev 27(1):73-83

7. Lorber J, Salfield S, Lonton T (1983) Isosorbide in the management of infantile hydrocephalus. Dev Med Child Neurol 25(4):502-511

8. Looney CB, Smith JK, Merck LH, Wolfe HM, Chescheir NC, Hamer RM et al (2007) Intracranial hemorrhage in asymptomatic neonates: prevalence on MR images and relationship to obstetric and neonatal risk factors. Radiology 242(2):535-541 
9. Rooks VJ, Eaton JP, Ruess L, Petermann GW, Keck-Wherley J, Pedersen RC (2008) Prevalence and evolution of intracranial hemorrhage in asymptomatic term infants. AJNR Am J Neuroradiol 29(6):1082-1089

10. Simonson C, Barlow P, Dehennin N, Sphel M, Toppet V, Murillo D et al (2007) Neonatal complications of vacuum-assisted delivery. Obstet Gynecol 109(3):626-633

11. Sandberg DI, Lamberti-Pasculli M, Drake JM, Humphreys RP, Rutka JT (2001) Spontaneous intraparenchymal hemorrhage in full-term neonates. Neurosurgery 48(5):1042-1048, discussion 1048-1049

12. Huang AH, Robertson RL (2004) Spontaneous superficial parenchymal and leptomeningeal hemorrhage in term neonates. AJNR Am J Neuroradiol 25(3):469-475

13. Slaughter L, Egelhoff J, Balmakund T (2009) Neurologic outcome in neonatal temporal lobe hemorrhagic venous infarcts. J Child Neurol 24(10):1236-1242

14. Hanigan WC, Powell FC, Palagallo G, Miller TC (1995) Lobar hemorrhages in full-term neonates. Child's Nerv Syst 11(5):276-280

15. Towner D, Castro MA, Eby-Wilkens E, Gilbert WM (1999) Effect of mode of delivery in nulliparous women on neonatal intracranial injury. N Engl J Med 341(23):1709-1714

16. Benedetti TJ (1999) Birth injury and method of delivery. N Engl J Med 341(23):1758-1759

17. Gardella C, Taylor M, Benedetti T, Hitti J, Critchlow C (2001) The effect of sequential use of vacuum and forceps for assisted vaginal delivery on neonatal and maternal outcomes. Am J Obstet Gynecol 185(4):896-902
18. Gupta SN, Kechli AM, Kanamalla US (2009) Intracranial hemorrhage in term newborns: management and outcomes. Pediatr Neurol 40(1):1-12

19. Jhawar BS, Ranger A, Steven D, Del Maestro RF (2003) Risk factors for intracranial hemorrhage among full-term infants: a case-control study. Neurosurgery 52(3):581-590, discussion 588590

20. Volpe JJ (2008) Intracranial hemorrhage: subdural, primary subarachnoid, cerebellar, intraventricular (term infant), and miscellaneous. In: Volpe JJ (ed) Neurology of the newborn, 5th edn. WB Saunders, Philadelphia, pp 481-588

21. Welch K, Strand R (1986) Traumatic parturitional intracranial hemorrhage. Dev Med Child Neurol 28(2):156-164

22. Kersbergen KJ, de Vries LS, van Straaten HL, Benders MJ, Nievelstein RA, Groenendaal F (2009) Anticoagulation therapy and imaging in neonates with a unilateral thalamic hemorrhage due to cerebral sinovenous thrombosis. Stroke 40(8):27542760

23. Ramenghi LA, Govaert P, Fumagalli M, Bassi L, Mosca F (2009) Neonatal cerebral sinovenous thrombosis. Semin Fetal Neonatal Med 14(5):278-283

24. Hoogstraate SR, Lequin MH, Huysman MA, Ahmed S, Govaert PP (2009) Apnoea in relation to neonatal temporal lobe haemorrhage. Eur J Paediatr Neurol 13(4):356-361

25. Sirsi D, Nadiminti L, Packard MA, Engel M, Solomon GE (2007) Apneic seizures: a sign of temporal lobe hemorrhage in full-term neonates. Pediatr Neurol Nov 37(5):366-370 